\title{
Effects of Prophylactic Laxatives for Opioid-Induced Constipation on Constipation Severity and Quality of Life among Patients with Cancer
}

\author{
Mohammad Alnaeem $^{1}{ }^{\circledR}$, Mohammad Ibrahim Al Qadire ${ }^{2}$, Jafar Alasad ${ }^{3 *}$ \\ ${ }^{1}$ Ministry of Health, Prince Hamzah Hospital, Amman, Jordan \\ ${ }^{2}$ Faculty of Nursing, Al Al-Bayt University, Mafraq, Jordan \\ ${ }^{3}$ Clinical Nursing Department, The University of Jordan, Amman, Jordan \\ Email:mmalnaeem33@gmail.com,mohammadqadire@aabu.edu.jo, ‘jalasad@ju.edu.jo
}

How to cite this paper: Alnaeem, M., $\mathrm{Al}$ Qadire, M.I. and Alasad, J. (2018) Effects of Prophylactic Laxatives for Opioid-Induced Constipation on Constipation Severity and Quality of Life among Patients with Cancer. Open Journal of Nursing, 8, 505-517. https://doi.org/10.4236/ojn.2018.88038

Received: June 9, 2018

Accepted: August 3, 2018

Published: August 6, 2018

Copyright (c) 2018 by authors and Scientific Research Publishing Inc. This work is licensed under the Creative Commons Attribution International License (CC BY 4.0).

http://creativecommons.org/licenses/by/4.0/

(c) (i) Open Access

\begin{abstract}
Background: To reduce patients' unnecessary suffering, Opioids Induced Constipation (OIC) should be identified and treated as early as possible with the most current treatment. Aims: to investigate the effectiveness of prophylactic laxatives in reducing the severity of OIC and its impact on cancer patients' quality of life. Methods: A Randomized Clinical Trial design was used. 57 cancer patients from one oncology clinic at a governmental hospital participated in the study. Patients in the intervention groups were given an oral colonic stimulant laxative (i.e. Bisacodyl, Dose $=3 \mathrm{tab} /$ day) or an oral colonic osmotic laxative (i.e. Lactulose, Dose $=15 \mathrm{ml}$ three times per day), while patients in the control continue receiving their routine care as usual. Results: A significant decrease in the severity of constipation symptoms among the intervention group at four weeks post-intervention was noted $(\mathrm{p}<0.001)$. In addition, there was a significant improvement in QOL among patients in the intervention group $(\mathrm{p}<0.001)$ and no significant difference in the control group $(\mathrm{p}=0.887)$. Conclusions: It can be concluded that the use prophylactic of first line laxatives concurrently with opioids decreased the severity of OIC and improved the QOL for cancer patients.
\end{abstract}

Keywords

Neoplasm, Adult, Opioids, Constipation, Stimulant, Laxatives

\section{Introduction}

Cancer is a complicated illness with multiple sequelae faced by patient and 
healthcare providers alike. Cancer, the second leading cause of death in Jordan, accounted for $16.4 \%$ of the recorded deaths in 2013 [1]. According to the Jordanian cancer registry, 8744 cases of cancer were discovered in 2013 [2]. Cancer patients suffer from a considerable number of symptoms [3] [4] [5] [6]. These symptoms may be as a result of cancer invasion or as a side effect of treatment [7]. Constipation is one of the most bothersome symptoms for cancer patients; it affects $40 \%$ of palliative care patients and up to $90 \%$ patients treated with opioids [8].

Most cancer patients receive opioid therapy to treat moderate to severe pain [9]. Opioids stimulate $\mu$-receptors inducing analgesia while peripherally acts on the gastrointestinal tract by suppression of acetylcholine freeing the myenteric plexus leading to relaxation of the longitudinal colon and muscle of the small intestine [10]. Consequently, it leads to a delay in intestinal or colonic transition by 1) decreased gastric emptying and propulsive movement 2) minimized gastric, biliary and pancreatic secretion and, 3) decreased water absorption from the bowel [11] [12]. As a result, these changes can lead to the following symptoms: loss of appetite, vomiting, constipation, abdominal discomfort, dry mouth, gastro-esophageal reflux, and anorexia, which is termed as Opioid-induced-constipation (OIC) [11] [13]. Unfortunately, the human body develops a tolerance for most of the opioid side effects with the except of constipation [14].

The prevalence of OIC among cancer patients is high, ranging from $30 \%$ to $50 \%$ [14] [15] [16]. Palliative care patients consider OIC as more upsetting and more difficult to endure than cancer pain itself [17]. Dhingra and colleagues (2013) conducted a study to evaluate the most common distressing symptoms among patients with advanced cancer; constipation was found to be more distressing than pain ( $18 \%, 6 \%$ respectively) [18].

There are many complications that result from OIC; stool impaction with excessive diarrhea, fecal incontinence, pseudo-occlusion of the bowel causing loss of appetite, nausea, and vomiting, in addition, urinary retention and incontinence, and insufficient absorption of drugs. All of these complications have a significant and negative affect on patients' quality of life [19]. Further, OIC may hinder pain treatment through the reduction or discontinuation of opioid use [20] [21]. To reduce patients' unnecessary suffering, OIC should be identified and treated as early as possible with the most current treatment available [22].

Laxatives remain the mainstay for the treatment of OIC. However, the use of laxatives in clinical practice is delayed and frequently inadequate [22]. A cohort study conducted by Skollerud, et al. (2013) among the Norwegian population who received opioids for cancer pain revealed that $44.1 \%$ of them were not given laxatives at any stage of treatment. They reported that only one fifth of cancer patients start taking laxatives with the first prescription of opioid medication. This indicates that the guidelines were not followed. In this regard, the authors suggested that prescribing laxatives should be initiated simultaneously with the opioid treatment as prophylaxis to prevent constipation in cancer patients [23]. In addition, another study found that most patients receiving long-standing 
opioid treatment require pharmacological therapy such as stool softeners, bowel stimulants, and bulk laxatives to maintain gastrointestinal motility [19].

Laxatives are considered the "gold standard" for management of OIC in cancer patients by inducing bowel movement and defecation [19] [20] [24]. Constipation risk factors should be frequently assessed and monitored [25]. If there are no contraindications laxatives should be initiated simultaneously with opioid therapy [13] [26] [27]. In contrast, if patients have an ileostomy or are complaining of chemotherapy-induced diarrhea, laxatives should be avoided [25].

The scarcity of prophylactic laxative use among cancer patients receiving opioids was associated with an increased risk of OIC, poor adherence to opioid therapy and deterioration in quality of life [18] [24] [28]. Studies conducted to assess OIC and to evaluate the effectiveness of prophylactic laxative management concluded that quality of life could be improved when OIC is managed early [16] [23]. For example, a study including 7836 participants with severe chronic pain treated with prophylactic laxatives revealed an improvement of $43 \%$ in overall quality of life after four weeks of management [29]. However, few randomized clinical trials evaluated the effectiveness of first line laxatives in treating OIC. This approach would increase our understanding of the role of laxatives in OIC treatment, strengthen the available research evidence, and reduce the suffering of cancer patients. Hence, the purpose of this study was to investigate the effectiveness of prophylactic laxatives in reducing the severity of OIC and its impact on cancer patients' quality of life.

\section{Aim}

The main aim of this study was to investigate the effectiveness of prophylactic laxatives in reducing the severity of OIC and its impact on cancer patients' quality of life.

\section{Research Hypotheses}

\section{Null Hypotheses}

1) The use of prophylactic laxatives has no effects on constipation severity among cancer patients receiving opioids.

2) The use of prophylactic laxatives has no impact on the quality of life of cancer patients receiving opioids.

\section{Alternative Hypotheses}

1) The use of prophylactic laxatives would reduce constipation severity among cancer patients receiving opioids.

2) The use of prophylactic laxatives would improve the quality of life of cancer patients receiving opioids.

\section{Materials and Methods}

\subsection{Design}

A Randomized Clinical trial design was used. 


\subsection{Sample and Sampling}

This study included 57 cancer patients who were conveniently recruited. Inclusion criteria for this study were as following: 18 years old or older, oriented, able to and provided consent, diagnosed with cancer and were receiving opioids without concurrent laxatives at the time of the study. Patients on an opioid antagonist, experiencing diarrhea or with an ileostomy, presently on concurrent treatment with laxatives, in the terminal phase of cancer, and who did not agree to participate in the study, were excluded.

\section{Sample size calculation}

The sample size was estimated by using the G-power software version 22 for the two-tailed independent $\mathrm{t}$-tests, based on the following parameters: large effect size of 0.8 , an alpha of 0.05 , and a power of 0.8 . A total number of 42 participants in the two groups were considered adequate. However, considering attrition, we recruited 69 patients. Throughout the study, twelve participants were dropped out, leaving 57 to complete. Five patients from control group and seven from the intervention group were dropped out for different reasons. These reasons include patients' death (control $=1$; intervention $=3$ ), adding laxative to treatment plan (control $=4$ ), and voluntary withdrawal from the study after consent (intervention $=4$ )

\subsection{Setting}

This study was conducted in a single oncology clinic at a governmental hospital in which approximately $26 \%$ of Jordanian cancer patients are treated.

\subsection{Instrumentation}

In this study, three questionnaires were used to collect the data: the first was the Confirmation of Constipation (CC) Scale. The CC scale was developed based on the Rome III criteria [30] to define constipation and to diagnose constipated participants. Per the scale, patients are constipated if they experienced at least two of the following symptoms: less than three stools per week; straining with at least $25 \%$ of stools; lumpy, hard stool at least $25 \%$ of the time; feeling of incomplete evacuation or sensation of blockage for at least $25 \%$ of stools and a need to manually remove stool at least $25 \%$ of the time. The CC scale consisted of six questions: Question 1 is "Have you been given laxatives during this period?" if the response was "yes", then the patient was requested to complete the CC; if the answer was "no", patient was excluded from participating in the study.

The second questionnaire was the Patient Assessment of Constipation Symptoms (PAC-SYM) tool which consists of 12-self-report items and was developed to measure the prevalence and intensity of OIC [31]. PAC-SYM is a reliable and valid measure of the presence and severity of opioid-induced constipation symptoms; the Cronbach $\alpha$ Coefficient for the scale is 0.80 [31]. The items are rated on a 5-point (0-4) Likert-type scale. Responses are scored as $0=$ absence of symptom, $1=$ mild, $2=$ moderate, $3=$ severe and $4=$ very severe. The mean of 
the total scores ranges from 0 to 4 , where lower scores reflect low symptom severity. This tool had already been translated into the Arabic language. In this study, the Cronbach $\alpha$ Coefficient was 0.97 which reflected excellent internal consistency of the scale.

The third instrument was the Patient Assessment of Constipation Quality of Life (PAC-QoL) tool [32]. It is a comprehensive and valid patient-reported assessment of the impact of constipation symptoms with an internal consistency of (Cronbach $\alpha$ Coefficient $=0.93$ ). Cronbach $\alpha$ Coefficient ranged from 0.81 to 0.91 for the sub-scales [32].

PAC-QoL is comprised of 28-items and addresses; constipation-related worries and concerns (11 items), physical discomfort (4 items), psychosocial discomfort (8 items), and satisfaction (5 items). These items are rated on a 5-point (0-4) Likert-type scale and the responses are scored as $0=$ "Not at all"/"None of the time"; 1 = "Quite a bit"/"Most of the time"; 2 = "Moderately"/"Some of the time"; 3 = "A little bit"/"A little of the time" and 4 = "Extremely"/"All of the time". Scores of items 18, 25 - 28 were reversed. The mean score is calculated by dividing the total score by the number of items for each participant. This resulted in a score ranging from 0 to 4 , and the lower score indicates better QOL. This questionnaire was translated into the Arabic language using a back-translation technique. In this study, the Cronbach $\alpha$ Coefficient was 0.98 which reflected a good internal consistency of scales. Cronbach $\alpha$ Coefficient from for the sub-scales ranged from 0.85 to 0.96 . In addition, sociodemographic and cancer-related data were collected about each participant.

\subsection{The Intervention}

The intervention in this study was the prophylactic laxatives which were prescribed by oncologists who provided a verbal agreement and commitment to participate in the study. The laxatives were provided as per the guidelines of Cancer Care Ontario Algorithm [33]; and Constipation Assessment \& Management Algorithm [34]. In this study, we used the recommended medications as the first line of treatment from both guidelines. This included an oral colonic stimulant laxative (i.e. Bisacodyl, Dose $=3$ tab/day) and an oral colonic osmotic laxative (i.e. Lactulose, Dose $=15 \mathrm{ml}$ three times per day), while patients in the control continue receiving their routine care as usual.

\subsection{Data Collection Procedure}

Data were collected between the 4 th of July and the 27 th of September 2016. As a first step, researchers approached the responsible nurses and informed them about the study aims, protocol and requirements. Then, the nurses were instructed to discuss the research project with patients that met the inclusion criteria. The patients that showed an interest in the study, were directly approached by one member of the research team. Thereafter, patients were given more details about the study, interventions, and the possible side effects (which are con- 
sidered minimal). After all questions were answered the interested participants were asked to sign the consent form.

All consented participants were asked to fill in the socio-demographic information and the Confirmation of Constipation (CC scale). Based on the CC scale, only constipated patients were enrolled and asked to complete baseline data including socio-demographic data, OIC severity and quality of life. Individuals were then randomly assigned into two groups; the intervention and the control group. Since the estimated sample size was less than 100, simple randomization technique was used. All patients with even medical record numbers were assigned to group A (intervention), and patients with odd medical record number assigned to group B (Control group). Patients in the intervention group received prophylactic laxatives prescribed by the assigned oncologist and patients in the control group continued their routine care and without prescribed prophylactic laxatives. Finally, after four weeks, follow-up data about OIC severity and quality of life was collected from both groups. During the four-week period, several phone calls were made to participants to answer questions and assure protocol adherence.

\subsection{Ethical Considerations}

Ethical approval to conduct the study was obtained from appropriate research committees. Complete information about the study purpose, protocol, possible side effects of laxative treatment and requirements were given to the participants before signing the consent form. In addition, all questions were answered and all study related information was attached to the consent form. Participants were given 24 hours to read and decide whether to participate or not. Participants were instructed to sign the consent form only after reading and completely understanding participation requirements. Further, ongoing information about laxatives and possible side effects were made available at any time through their primary care physician. All participants were assured that they have the right to withdraw from the study at any time for any reason without any effect on their medical or nursing care.

\subsection{Data Analysis}

Data analysis was performed using the Statistical Package for the Social Sciences version 17 software program (SPSS, Chicago, IL, USA). Descriptive and inferential analyses of the demographic and major study variables were conducted. For continuous variables, the mean and standard deviation were described and rounded to one decimal. For categorical variables, the number and percent distribution by category were described Independent t-test was conducted to compare the differences in OIC severity and QOL for the intervention and control group. The difference between the baseline and four weeks post-intervention in OIC severity and QOL for each group was conducted using a paired t-test. 


\section{Results}

\subsection{Description of Sample Demographics}

A total number of 57 cancer patients participated in the study (control $=30$, Intervention $=27$ ). The mean age of all participants was 53.0 SD 10.9 years (control $=52.5$ SD 9.2, Intervention $=53.7$ SD 12.4). Sixty percent $(n=35)$ of all patients were diagnosed with cancer within the last year. Approximately one in six patients reported that their previous bowel movement was more than three days ago. Table 1 details sample characteristics in the both groups.

\subsection{Constipation Severity}

At the start of the study participants in both group had several constipation symptoms. The mean total score on the PAC-SYM scale was 18.5 SD 5.77 for the intervention group and 15.3 SD 6.10 for the control group. At the follow up, the mean constipation symptoms scale was reduced to $9.22 \mathrm{SD}=7.13$ in the intervention group, minimal change was noted in the control group was 14.9 ( $\mathrm{SD}=$ 9.77). The independent $t$-test showed no significant difference in the mean score of PAC-SYM between intervention and control groups at baseline $(\mathrm{t}=1.99, \mathrm{df}=$ $55, p=0.051)$. While at post-intervention, a significant difference in the mean of constipation symptom scale was found $(t=2.47, \mathrm{df}=55, \mathrm{p}=0.017)$.

Furthermore, paired t-test was conducted to compare the mean of total constipation symptom scale for the same group at baseline and at four weeks post-intervention. A significant decrease in the severity of constipation symptoms among the intervention group at four weeks post-intervention was noted $(\mathrm{p}<0.001)$. The decrease in the control group was minimal and not statistically significant (see Table 2).

\subsection{Quality of Life}

The QOL was conducted at baseline and four weeks post-intervention. Although, a significant difference in QOL between intervention and control groups was found at baseline, no significant difference in QOL was observed at four weeks post-intervention. The $\mathrm{t}$-test was performed at baseline (intervention: $\mathrm{M}=$ 47.8, $\mathrm{SD}=23.2$; control: $\mathrm{M}=35.4, \mathrm{SD}=22.2$ ) and four weeks post-intervention (intervention: $\mathrm{M}=37.9, \mathrm{SD}=22.8$; control: $\mathrm{M}=34.9, \mathrm{SD}=25.5$ ). The paired-t-test was used to compare the means of the QOL for the same group at baseline and at four weeks post-intervention. Results revealed a significant improvement in QOL among patients in the intervention group $(\mathrm{t}=4.21, \mathrm{df}=26, \mathrm{p}$ $<0.001)$ and no significant difference in the control group $(\mathrm{t}=0.14, \mathrm{df}=26, \mathrm{p}=$ $0.887)$ see Table 3.

\section{Discussion}

As demonstrated by this study, prophylactic first line laxatives (i.e. Bisacodyl, Dose $=3 \mathrm{tab} /$ day or Lactulose, Dose $=15 \mathrm{ml}$ three times per day) should be given concurrently with opioid administration. This apporach was effective in re 
Table 1. Demographic characteristic of participants.

\begin{tabular}{|c|c|c|}
\hline & \multicolumn{2}{|c|}{ Frequency (\%) } \\
\hline & Intervention $(n=27)$ & Control $(n=30)$ \\
\hline \multicolumn{3}{|l|}{ Gender } \\
\hline Male & $14(51.8)$ & $11(36.7)$ \\
\hline Female & $13(48.2)$ & $19(63.3)$ \\
\hline \multicolumn{3}{|l|}{ Marital status } \\
\hline Single & $2(7.4)$ & $2(6.7)$ \\
\hline Married & $23(85.2)$ & $22(73.3)$ \\
\hline Divorced or widowed & $2(7.4)$ & $6(20.0)$ \\
\hline \multicolumn{3}{|l|}{ Cancer primary site } \\
\hline Breast & $4(14.8)$ & $2(6.7)$ \\
\hline Lung and nasopharyngeal & $5(18.5)$ & $5(16.7)$ \\
\hline Prostate & $6(22.2)$ & $1(3.3)$ \\
\hline Uterus \& ovarian & $1(3.7)$ & $4(13.3)$ \\
\hline Gastrointestinal & $7(25.9)$ & $3(9.9)$ \\
\hline Others & $4(14.8)$ & $7(23.4)$ \\
\hline \multicolumn{3}{|l|}{ Duration of cancer diagnosis } \\
\hline Less than one year & $15(55.6)$ & $12(43.3)$ \\
\hline One year & $3(11.1)$ & $4(13.3)$ \\
\hline More than one year & $9(33.3)$ & $13(43.4)$ \\
\hline \multicolumn{3}{|l|}{ Religion } \\
\hline Islam & $26(96.3)$ & $29(96.7)$ \\
\hline Christine & $1(3.7)$ & $1(3.3)$ \\
\hline \multicolumn{3}{|l|}{ Opioids Therapy } \\
\hline Tramadol & $19(70.4)$ & $25(83.3)$ \\
\hline Morphine & $8(29.6)$ & $5(16.7)$ \\
\hline
\end{tabular}

Table 2. Within-group analysis of constipation symptoms severity at baseline and post-intervention.

\begin{tabular}{ccccccc}
\hline \multicolumn{7}{c}{ Severity of constipation } \\
& \multicolumn{1}{c}{ Before intervention } & Post-intervention & & & p-value \\
\cline { 2 - 7 } & $\mathrm{M}$ & $\mathrm{SD}$ & $\mathrm{M}$ & $\mathrm{SD}$ & $\mathrm{t}$ & \\
\hline Intervention & 18.5 & 5.77 & 9.22 & 7.13 & 8.99 & $<0.001^{*}$ \\
Control & 15.3 & 6.10 & 14.9 & 9.77 & 2.47 & 0.801 \\
\hline
\end{tabular}

${ }^{*}$ Significant: $\mathrm{p}<0.001$.

ducting constipation severity and improving quality of life for cancer patients. In this study, the majority of patients in both groups were constipated (73.7\%) at baseline. This finding is consistent with several other similar studies. Bell, et al. (2007) reported that $74 \%$ - $92 \%$ of cancer patients treated with opioid without laxatives had mild to moderate constipation. Cancer patients receiving prophylactic 
Table 3. Within group analysis of quality of life score pre-and post intervention.

\begin{tabular}{cccccccc}
\hline & \multicolumn{7}{c}{ Quality of life } \\
& $\begin{array}{c}\text { Before } \\
\text { intervention }\end{array}$ & \multicolumn{5}{c}{ Post-intervention } \\
\cline { 2 - 7 } & $\mathrm{M}$ & $\mathrm{SD}$ & $\mathrm{M}$ & $\mathrm{SD}$ & $\mathrm{t}$ & $\mathrm{p}$ \\
\hline Intervention & 47.7 & 23.2 & 37.9 & 22.8 & 4.21 & $0.01^{*}$ \\
Control & 35.4 & 22.2 & 34.9 & 25.5 & 0.14 & 0.887 \\
\hline
\end{tabular}

${ }^{*}$ Significant: $\mathrm{p}<0.001$.

laxatives with opioids had a $34 \%$ reduction in OIC when compared to patients not receiving prophylactic laxatives with opioid treatment [35]. Thus, the incidence of OIC may be expected to increase among cancer patients who do not have prescribed laxatives concurrent with opioid use. Strategies must be put into place that prevent or manage OIC, such as safe and effective first line laxatives.

The findings in this study reported a reduction in constipation severity at four weeks post-intervention among the intervention group compared to control group where no improvement was noted. This indicates that the use of prophylactic laxatives was effective in reducing the constipation occurrence when used at the start of opioid therapy. This finding is congruent with research conducted to evaluate effectiveness of prophylactic laxatives for constipation in critically ill patients. The results revealed that the patients in the intervention group had a bowel movement far more frequently than the patients in the control group, concluding the effectiveness of prophylactic laxative use in constipation prevention [36]. Moreover, two randomized controlled trials, one of the two assessed the effect of Bisacodyl (BIS) on patients with chronic constipation, $(n=736)$ compared to Sodium Picosulfate (SPS) $(n=468)$ for chronic constipation. The pooled analysis of data from the two randomized controlled trials compared the patients in intervention group 468 (who received SPS/BIS); with control group 250 (who received placebo) revealed that the number of spontaneous bowel movements per week during the trials was notably raised over four weeks from baseline among intervention groups more than control group [37].

Increasing the awareness of laxative use concurrent with the opioid therapy could be effective in increasing the adherence to the recommended guidelines on the treatment of OIC. This can be achieved through providing educational intervention for physicians and nurses regarding assessment, prevention and management of OIC. However, such intervention needs to be tested for its effectiveness and feasibility in clinical practice settings. Further, knowledgeable physicians and nurses are considered a primary source of information for patients, thus, they must teach patients about the importance of laxative use in preventing the occurrence and the treatment of OIC. Patients would likely cooperate and adhere to the treatment plan if they experience a reduction in treatment complications such as constipation.

Regarding quality of life, cancer patients who were prescribed prophylactic 
laxatives (intervention group) had a significant improvement in QOL at four weeks post-intervention in comparison to patients in the control group. This finding is consistent with the findings of previous studies [37]. It was reported that patients in the intervention groups had a significant improvement in overall score of QOL compared to a placebo group, (47\%, $14.5 \%$ respectively) [37]. Further, a survey was conducted on a sample of cancer patients from UK, Cana$\mathrm{da}$, and Germany to evaluate the magnitude of OIC [16]. It was found that OIC was prevalent and quality of life was severely compromised. However, most of patients acknowledged a reduction on OIC and improvement in quality of life after the laxative therapy was initiated [16].

Quality of life is paramount in cancer care, a previous study reported high prevalence of cancer related symptoms, including OIC, among Jordanian cancer patients and poor quality of life [6]. Hence, a conservative approach for treatment of OIC in cancer patients would contribute to better quality of life. However, the use of more than one laxative and for longer period found to be correlated with low quality of life [38].

The results of this study should be interpreted in the light of the following limitations. First, study participants were recruited from a single setting, OIC assessment and management might be different at different settings and thus the generalization of the findings might be restricted to the place of the study. Second, the constipation severity was assessed through an instrument that is self-reported which may result in over or under-estimation of the symptoms or the effects of the given treatment. Using another objective assessment such as number of actual bowel movement is recommended.

\section{Key Points for Practice and Research}

The results of this study have the following implications for nursing practice. First, nurses working with cancer patients should consider a comprehensive assessment of OIC as early as possible as it one of prevalent and devastating symptom. Second, nurses and based on their assessment should advocate the use of laxative as prophylactic in anticipation of OIC occurrence. Finally, nurses are in unique position to improve the management of OIC as they are a crucial component of the healthcare team and they spend the longest time of the patients. On research tract, an educational intervention for physicians and nurses regarding assessment, prevention and management of OIC needs to be tested for its effectiveness and feasibility in clinical practice settings.

\section{Conclusion}

This study evaluated the effects of prophylactic laxatives on constipation severity and QOL among cancer patients. Regardless of severity, most cancer patients have experienced OIC and have a low quality of life mean score. It can be concluded that the use prophylactic of first line laxatives concurrently with opioids decreased the severity of OIC and improved the QOL for cancer patients. Hence, 
the use of prophylactic laxatives is recommended to be prescribed once the opioids treatment is initiated.

\section{Acknowledgements}

The authors would like to thank the University of Jordan for partially funding the study.

\section{Conflicts of Interest}

The authors declare no conflicts of interest regarding the publication of this paper.

\section{References}

[1] Ministry of Health (2017) Mortality Data in Jordan, 2013. http://www.moh.gov.jo/Pages/viewpage.aspx?pageID $=248$

[2] Jordan Cancer Registry (2017) Cancer Incidence in Jordan-2013, Ministry of Health Jordan.

[3] Abernethy, A.P., Wheeler, J.L. and Zafar, S.Y. (2009) Detailing of Gastrointestinal Symptoms in Cancer Patients with Advanced Disease: New Methodologies, New Insights, and a Proposed Approach. Current Opinion in Supportive and Palliative Care, 3, 41-49. https://doi.org/10.1097/SPC.0b013e32832531ce

[4] Desai, K., et al. (2013) Prevalence and Risk Factors for Insomnia among Breast Cancer Patients on Aromatase Inhibitors. Supportive Care in Cancer, 21, 43-51. https://doi.org/10.1007/s00520-012-1490-Z

[5] Ho, S.-Y., et al. (2015) A Longitudinal Study of Depression, Fatigue, and Sleep Disturbances as a Symptom Cluster in Women with Breast Cancer. Journal of Pain and Symptom Management, 49, 707-715. https://doi.org/10.1016/j.jpainsymman.2014.09.009

[6] Al Qadire, M. and Al Khalaileh, M. (2016) Prevalence of Symptoms and Quality of Life among Jordanian Cancer Patients. Clinical Nursing Research, 25, 174-191. https://doi.org/10.1177/1054773814564212

[7] Yarbro, C.H., Wujcik, D. and Gobel, B.H. (2010) Cancer Nursing: Principles and Practice. Jones \& Bartlett Publishers, Burlington.

[8] Rhondali, W., Nguyen, L., Palmer, L., Kang, D.-H., David Hui, R.N. and Bruera, E. (2013) Self-Reported Constipation in Patients with Advanced Cancer: A Preliminary Report. Journal of Pain and Symptom Management, 45, 23-32. https://doi.org/10.1016/j.jpainsymman.2012.01.009

[9] Deibert, P., Xander, C., Blum, H.E. and Becker, G. (2009) Methylnaltrexone: The Evidence for Its Use in the Management of Opioid-Induced Constipation. Core Evidence, 4, 247-258. https://doi.org/10.2147/CE.S8556

[10] Kumar, L., Barker, C. and Emmanuel, A. (2014) Opioid-Induced Constipation: Pathophysiology, Clinical Consequences, and Management. Gastroenterology Research and Practice, 2014, Article ID: 141737.

[11] De Schepper, H., et al. (2004) Opioids and the Gut: Pharmacology and Current Clinical Experience. Neurogastroenterology \& Motility, 16, 383-394. https://doi.org/10.1111/j.1365-2982.2004.00513.x

[12] Galligan, J.J. and Akbarali, H.I. (2014) Molecular Physiology of Enteric Opioid Re- 
ceptors. The American Journal of Gastroenterology Supplements, 2, 17-21. https://doi.org/10.1038/ajgsup.2014.5

[13] Brock, C., et al. (2012) Opioid-Induced Bowel Dysfunction. Drugs, 72, 1847-1865. https://doi.org/10.2165/11634970-000000000-00000

[14] Vallerand, A.H. (2017) Opioid-Induced Constipation. The Journal for Nurse Practitioners, 13, 170-174.

[15] Erichsén, E., Milberg, A., Jaarsma, T. and Friedrichsen, M.J. (2015) Constipation in Specialized Palliative Care: Prevalence, Definition, and Patient-Perceived Symptom Distress. Journal of Palliative Medicine, 18, 585-592.

https://doi.org/10.1089/jpm.2014.0414

[16] Coyne, K.S., Sexton, C., LoCasale, R.J., King, F.R., Margolis, M.K. and Ahmedzai, S.H. (2016) Opioid-Induced Constipation among a Convenience Sample of Patients with Cancer Pain. Frontiers in Oncology, 6, 131. https://doi.org/10.3389/fonc.2016.00131

[17] Larkin, P., et al. (2008) The Management of Constipation in Palliative Care: Clinical Practice Recommendations. Palliative Medicine, 22, 796-807. https://doi.org/10.1177/0269216308096908

[18] Dhingra, L., et al. (2013) A Qualitative Study to Explore Psychological Distress and Illness Burden Associated with Opioid-Induced Constipation in Cancer Patients with Advanced Disease. Palliative Medicine, 27, 447-456. https://doi.org/10.1177/0269216312450358

[19] Reimer, K., et al. (2009) Meeting the Challenges of Opioid-Induced Constipation in Chronic Pain Management-A Novel Approach. Pharmacology, 83, 10-17. https://doi.org/10.1159/000165778

[20] Bell, T.J., et al. (2009) The Prevalence, Severity, and Impact of Opioid-Induced Bowel Dysfunction: Results of a US and European Patient Survey (PROBE 1). Pain Medicine, 10, 35-42. https://doi.org/10.1111/j.1526-4637.2008.00495.x

[21] Becker, G., Galandi, D. and Blum, H.E. (2007) Peripherally Acting Opioid Antagonists in the Treatment of Opiate-Related Constipation: A Systematic Review. Journal of Pain and Symptom Management, 34, 547-565. https://doi.org/10.1016/j.jpainsymman.2006.12.018

[22] Serra, J., Mascort-Roca, J., Marzo-Castillejo, M., et al. (2017) Clinical Practice Guidelines for the Management of Constipation in Adults. Part 2: Diagnosis and Treatment. Gastroenterologia y Hepatologia, 40, 303-316.

[23] Skollerud, L.M., et al. (2013) Laxative Prescriptions to Cancer Outpatients Receiving Opioids: A Study from the Norwegian Prescription Database. Supportive Care in Cancer, 21, 67-73. https://doi.org/10.1007/s00520-012-1494-8

[24] Clemens, K.E. and Klaschik, E. (2008) Management of Constipation in Palliative Care Patients. Current Opinion in Supportive and Palliative Care, 2, 22-27. https://doi.org/10.1097/SPC.0b013e3282f53146

[25] Wein, S. (2012) Opioid-Induced Constipation. Journal of Pain \& Palliative Care Pharmacotherapy, 26, 382-384. https://doi.org/10.3109/15360288.2012.734907

[26] Diego, L., et al. (2011) Novel Opioid Antagonists for Opioid-Induced Bowel Dysfunction. Expert Opinion on Investigational Drugs, 20, 1047-1056. https://doi.org/10.1517/13543784.2011.592830

[27] Osterbrink, J. and Haas, U. (2008) Opioid-Induced Bowel Dysfunction: A Literature Analysis on Pathophysiology and Treatment. Wiener Medizinische Wochenschrift (1946), 158, 621-626. 
[28] Ishihara, M., et al. (2010) Pharmaceutical Interventions Facilitate Premedication and Prevent Opioid-Induced Constipation and Emesis in Cancer Patients. Supportive Care in Cancer, 18, 1531-1538. https://doi.org/10.1007/s00520-009-0775-3

[29] Nolte, T., Schutter, U. and Loewenstein, O. (2014) Cancer Pain Therapy with a Fixed Combination of Prolonged-Release Oxycodone/Naloxone: Results from a Non-Interventional Study. Pragmatic and Observational Research, 5, 1.

[30] Drossman, D. (2007) Introduction. The Rome Foundation and Rome III. Neurogastroenterology \& Motility, 19, 783-786. https://doi.org/10.1111/j.1365-2982.2007.01001.x

[31] Frank, L., Kleinman, L., Farup, C., Taylor, L. and Miner, P. (1999) Psychometric Validation of a Constipation Symptom Assessment Questionnaire. Scandinavian Journal of Gastroenterology, 34, 870-877. https://doi.org/10.1080/003655299750025327

[32] Marquis, P., et al. (2005) Development and Validation of the Patient Assessment of Constipation Quality of Life Questionnaire. Scandinavian Journal of Gastroenterology, 40, 540-551. https://doi.org/10.1080/00365520510012208

[33] Cancer Care Ontario's Symptom Management Group (2012) Symptom \& Side Effect Management. https://www.cancercareontario.ca/en/symptom-management

[34] Winnipeg Regional Health Authority (WRHA) Palliative Care Program (2012) WRHA Palliative Care Program Constipation Assessment \& Management Algorithm.

http://www.virtualhospice.ca/Assets/WRHA\%20Constipation\%20Assessment\%20\& \%20\%20Management\%20Algorithm Final\%20August\%201 20130318165638.pdf

[35] Ishihara, M., et al. (2012) A Multi-institutional Study Analyzing Effect of Prophylactic Medication for Prevention of Opioid-induced Gastrointestinal Dysfunction. The Clinical Journal of Pain, 28, 373-381. https://doi.org/10.1097/AJP.0b013e318237d626

[36] Masri, Y., Baker, J. and Ahmed, R. (2010) Prophylactic Use of Laxative for Constipation in Critically Ill Patients. Critical Care, 14, P534.

https://doi.org/10.1186/cc8766

[37] Müller-Lissner, S., et al. (2017) Bisacodyl and Sodium Picosulfate Improve Bowel Function and Quality of Life in Patients with Chronic Constipation-Analysis of Pooled Data from Two Randomized Controlled Trials. Open Journal of Gastroenterology, 7, 32.

[38] Christensen, H.N., et al. (2016) Opioid-İnduced Constipation, Use of Laxatives, and Health-Related Quality of Life. Scandinavian Journal of Pain, 11, 104-110. https://doi.org/10.1016/j.sjpain.2015.12.007 